\section{SYNGAMIASIS IN PEAFOWL (PAVo CRISTATUS)}

\author{
K.S.Subramanian \\ Veterinary University Training and Research Centre, Rajapalayam, \\ Tamil Nadu 626117, India. \\ e-mail:drkswildlifevet@yahoo.co.in
}

\begin{abstract}
Although the Peafowl (Pavo cristatus) is commonly seen in India and Sri Lanka, documentation on health-related studies are very scanty. Syngamiasis or gape worm infection has been reported in chicken, Turkey, Guinea Fowl, kestrels and pheasants (Wallach \& Boever, 1983). This study documents the incidence of Syngamus trachea in a free-ranging Peafowl.
\end{abstract}

\section{Materials and methods}

A carcass of a peacock belonging to Deer Park, Tirunelveli was subjected to postmortem examination. On examination of the tracheal region, small, bright red-coloured worms attached to the blood-streaked mucosal surface of the trachea were observed.

The worms collected were washed in distilled water and cleared using ascending grades of alcohol and finally in carbolic acid for two minutes. The worms mounted in d.p.x. solution were identified and measured. Faecal samples were collected and screened by sedimentation technique (Soulsby, 1982) for the presence of parasitic ova. Morphometry of the parasitic ova were also measured by using an ocular eyepiece scale calibrated by means of a stage micometer.

The faecal samples revealed the presence of ova of Syngamus sp. Soulsby (1982) reported that the Syngamus ova from fowl, Turkey, Guinea Fowl and pheasants were measured to be 70$100 \times 43-46 \mu$ in size, where as in the present study, the mean morphometry of Syngamus ova was found to be $50 \times 30 \mu$, which might be specific to Peafowl. The worms collected from the trachea of the peacock were identified as Syngamus trachea (Gape Worm) based on its anatomical location and the characteristic appearance of ' $y$ ' shaped structure of the worms where in the male and female are found to be in permanent copulation (Soulsby, 1982).

The mean length of male and female worms were measured to be $2.5 \mathrm{~mm}$ and $13.5 \mathrm{~mm}$ respectively. Soulsby (1982) reported that the length of male and female worms recovered from turkeys, pheasants and various wild birds were measured to be of 2-6mm and 5-20mm respectively which coincided with the observations of the present study. Gardiner (1996) stated that syngamiasis was one of the common parasitic problems encountered in Peafowl in which the infected bird stretches its neck making gasping movements. Infection in Peafowl occurs by consuming earthworms which act as intermediate host for the Gape Worm.

\section{Acknowledgement}

The author thanks the Tamil Nadu Forest Department and TANUVAS, Chennai for the facilities provided.

\section{References}

Gardiner, T.P. (1996). Peafowl - Their Conservation, Breeding and Management. World pheasant association, U.K. 45pp.

Soulsby, E.J.L. (1982). Helminths, Arthropods and Protozoa of Domesticated Animals, pp.196-197. $7^{\text {th }}$ ed. Elbs, London.

Wallach, D.J. and W.J. Boever (1983). Diseases of Exotic Animals. W.B. Saunders Company, London. 947pp. 